\title{
Cholesteryl ester transfer protein: ace of spades, queen of hearts, or the joker?
}

\author{
Norman E. Miller * \\ Magdalen College, Oxford University, Oxford, UK
}

Keywords: cholesteryl ester transfer protein, CETP inhibitors, HDL, cardiovascular diseases, atherosclerosis

\section{OPEN ACCESS}

Edited by:

Chiranjib Chakraborty,

Galgotias University, India

Reviewed by:

Ghanshyam Upadhyay,

City College of New York-CUNY, USA

${ }^{*}$ Correspondence:

Norman E. Miller,

n.e.miller@btinternet.com

Specialty section:

This article was submitted to

Experimental Pharmacology and Drug

Discovery,

a section of the journal

Frontiers in Pharmacology

Received: 05 June 2015

Accepted: 29 June 2015

Published: 14 July 2015

Citation:

Miller NE (2015) Cholesteryl ester transfer protein: ace of spades, queen

of hearts, or the joker?

Front. Pharmacol. 6:145.

doi: 10.3389/fphar.2015.00145
Plasma cholesteryl ester transfer protein (CETP) catalyzes the transfer of CEs from high-density lipoproteins (HDLs) to triglyceride-rich and low-density lipoproteins (LDLs). The hypothesis that CETP inhibition will prevent cardiovascular disease (CVD) was based on the fact that low activity increases HDL cholesterol and decreases LDL cholesterol. Early reports that CETP gene transfer increased atherosclerosis in mice, and that CETP inhibition reduced lesions in cholesterol-fed rabbits fuelled enthusiasm for the approach. Although some cautioned that the development of CETP inhibitors was premature owing to uncertainties about their impact on the remodeling of HDLs and reverse cholesterol transport (RCT) in humans (Fielding and Havel, 1996), drug discovery programmes proceeded. Two parallel research tracks then emerged. While industry progressed to clinical studies, academics sought to clarify the relations of CETP activity to RCT and atherosclerosis. Two drugs reached Phase 3 clinical trials. However, ILLUMINATE was terminated when torcetrapib was found to have had increased CVD. Five years later, Dal-OUTCOMES was aborted when it was evident dalcetrapib was not conferring any benefit.

After reviewing the literature up to May 2014, Miller (2014) concluded that CETP inhibition is more likely to increase CVD than prevent it, and was of the opinion that ongoing trials should be stopped. Since then several further pertinent studies have been published. They include four meta-analyses of the Taq1B polymorphism of the CETP gene. Cao et al. (2014) concluded that B2B2 homozygotes (low CETP activity, high HDL cholesterol) have a lower risk of myocardial infarction (MI) than B1B1 subjects. Using Mendelian randomization, Wu et al. (2014) found the B2 allele to be associated with a lower risk of coronary heart disease (CHD). However, another Mendelian randomization (Niu and Qi, 2015) found the B2 allele to be associated with a higher risk of CVD than the B1 allele. In this context, an earlier meta-analysis by Dullaart and Sluiter (2008) is of interest. These authors found that B2B2 carriers were less frequent among cases drawn from samples at high CVD risk than among cases drawn from population-based samples. Furthermore, in the latter case the odds ratio for CVD was 1.45 (95\% CI: 1.07-1.95) in B2B2 relative to B1B1, while in the former it was 0.84 (0.74-0.96), suggesting that in the general population the $\mathrm{B} 2$ allele is actually associated with higher CVD risk in spite of the higher HDL cholesterol. Regieli et al. (2008) had come to a similar conclusion in the REGRESS study. After following 812 men with CHD on statins for 10 years, the $\mathrm{B} 2$ allele was associated with a hazard ratio for CVD death of $1.59(P=0.01)$ despite the expected low CETP activity and high HDL cholesterol.

Four additional meta-analyses looked at the impact of CETP inhibitors on CVD risk as part of larger studies of the effects of HDL cholesterol-raising agents in patients taking statins. All four concluded that the trials have not demonstrated a beneficial effect (Keene et al., 2014; Hourcade-Potelleret et al., 2015; Ip et al., 2015; Verdoia et al., 2015). In clinical studies, Gu et al. (2014) reported that although the A allele of the $-629^{\circ} \mathrm{C} / \mathrm{A}$ polymorphism of the CETP gene was associated with a lower plasma CETP concentration than the $\mathrm{C}$ allele, it had not reduced CVD events in patients taking atorvastatin. Kastelein et al. (2015) observed no significant effect of anacetrapib on CVD incidence (four events vs. zero in the treated and placebo groups, respectively) 
during 12 months of follow-up in patients with heterozygous familial hypercholesterolemia already on lipid-lowering treatment. Scharnagl et al. (2014) confirmed earlier reports that human plasma samples with low CETP concentrations were less effective in promoting cholesterol efflux from cultured macrophages than samples with high concentrations. In animal studies, Kühnast et al. (2015) found that anacetrapib reduced atherosclerosis in $\mathrm{APOE}^{\star} 3$ Leiden.CETP transgenic mice. Briand et al. (2014) compared anacetrapib with dalcetrapib in hamsters, a species with natural CETP. In normal animals, neither drug at doses equipotent for CETP inhibition (by 60\%) had any effect on macrophage-to-feces RCT, although they did lower equally the fractional clearance rate of HDL-CE. In dyslipidaemic animals, anacetrapib increased RCT, whereas an equipotent dose of dalcetrapib reduced it. Liu et al. (2015) found that inhibition of DNA topoisomerase II (Topo II) by etoposide, tenipooside or Topo II siRNA increased CETP gene expression and CETP secretion in HepG 2 cells. When given to CETP transgenic mice, teniposide induced CETP expression in the liver, and increased macrophage-to-feces RCT to a greater degree than in wild-type mice with no CETP. Using a computer model of lipoprotein metabolism to analyze the on/off kinetics of the short-acting potent CETP inhibitor RG7232, Lu et al. (2015) concluded that inhibition of CETP is likely to reduce prebeta HDL production in humans.

During the past 25 years, lipidologists have become accustomed to the controversies in this field. While the decrease in LDL cholesterol has been assumed to be beneficial, the rise in HDL cholesterol has long prompted discussion on three fronts. First, the early concerns (Fielding and Havel, 1996) that HDL remodeling might be deranged, leading to lowered prebeta HDL production, have not been allayed. Second, the early assumption that the rise in HDL CE would increase the direct delivery of CEs to the liver via SR-B1 receptors was challenged by evidence that the receptors may be saturated at normal plasma HDL concentrations (Woollett and Spady, 1997; Nieland et al., 2011). Third, evidence was reported that the large CE-rich HDLs produced by CETP inhibition might be dysfunctional. Despite

\section{References}

Briand, F., Theiblemont, O., Muzotte, E., Burr, N., Urbain, I., Sulpice, T., et al. (2014). Anacetrapib and dalcetrapib differentially alter HDL metabolism and macrophage-to-feces reverse cholesterol transport at similar levels of CETP inhibition in hamsters. Eur. J. Pharmacol. 740, 135-143. doi: 10.1016/j.ejphar.2014.06.022

Cao, M., Zhou, Z. W., Fang, B. J., Zhao, C. G., and Zhou, D. (2014). Meta-analysis of cholesteryl ester transfer protein Taq1B polymorphism and risk of myocardial infarction. Medicine (Baltimore) 93:e160. doi: 10.1097/MD.0000000000000160

Corsetti, J. P., Gansevoort, R. T., Navis, G., Sparks, C. E., and Dullaart, R. P. (2011). LPL polymorphism (D9N) predicts cardiovascular disease risk directly and through interaction with CETP polymorphism (TaqIB) in women with high HDL cholesterol and CRP. Atherosclerosis 214, 373-376. doi: 10.1016/j.atherosclerosis.2010.11.029

Dullaart, R. P., and Sluiter, W. J. (2008). Common variation in the CETP gene and the implications for cardiovascular disease and its treatment: an updated analysis. Pharmacogenomics 9, 747-763. doi: 10.2217/14622416.9.6.747 these concerns, momentum was maintained in the hope that the reduction of LDL would more than offset any adverse effects on HDL. However, as time has gone on the cumulative evidence has increasingly pointed to CETP having both a facilitative role in RCT and a net preventative effect on CVD. The recent work summarized above has not weakened this evidence.

Although the debate has centered largely on whether CETP inhibition is likely to have a beneficial or detrimental impact on CVD, the possibility that the net effect of the combined changes in LDL and HDL metabolism might vary according to the prevailing physiologic conditions, and therefore from subject to subject, and from time to time in the same subject, also merits consideration. Being at a crossroad in the transport of lipids, and having multiple effects on lipoprotein metabolism, the overall effects of CETP activity and its inhibition might vary according to the ambient HDL and LDL particle concentrations, for example, or to interplay with other genes. Reports of interactions between CETP alleles and alleles of the lipoprotein lipase (Corsetti et al., 2011), hepatic lipase (Soyal et al., 2011), apolipoprotein E (Sun et al., 2014), and nitric oxide synthase (Rahimi et al., 2012) genes seem to add weight to this possibility.

It remains that the tandem hypotheses that CETP inhibition will both enhance RCT and prevent CVD are without sound scientific bases. Indeed a growing body of evidence of three kinds now supports the contrary. First, in three out of three experimental studies of their type, macrophage-to-feces RCT in vivo was increased when CETP expression was enhanced (Briand et al., 2014; Miller, 2014). Second, each of five studies that examined the effect of CETP activity in human plasma on cholesterol efflux from cultured cells in vitro found that high activity plasma was more effective than low activity (Miller, 2014; Scharnagl et al., 2014). Third, each of six prospective cohort observational studies have found that CETP concentration or activity was related inversely to CVD incidence (Miller, 2014). In the author's opinion, this is sufficient to justify stopping all ongoing clinical trials of CETP inhibitors. Carrying on and hoping for the best, while participants are unaware of the evolving evidence base, is not acceptable.
Fielding, C. J., and Havel, R. J. (1996). Cholesteryl ester transfer protein-friend or foe? J. Clin. Invest. 97, 267-278. doi: 10.1172/JCI118719

Gu, G. L., Xu, X.-L., Yang, Q.-Y., and Zeng, R.-L. (2014). Effect of CETP polymorphism on atorvastatin lipid-regulating effect and clinical prognosis of patients with coronary heart disease. Med. Sci. Monit. 20, 2824-2829. doi: 10.12659/MSM.892711

Hourcade-Potelleret, F., Laporte, S., Lehnert, V., Delmar, P., Benghozi, R. Torriani, U., et al. (2015). Clinical benefit from pharmacological elevation of high-density lipoprotein cholesterol: meta-regression analysis. Heart 101, 847-853. doi: 10.1136/heartjnl-2014-306691

Ip, C. K., Jin, D. M., Gao, J. J., Meng, Z., Tan, Z., Wang, J. F., et al. (2015). Effects of add-on lipid-modifying therapy on top of background statin treatment on major cardiovascular events: a meta-analysis of randomized controlled trials. Int. J. Cardiol. 191, 138-148. doi: 10.1016/j.ijcard.2015.04.228

Kastelein, J. J., Besseling, J., Shah, S., Bergeron, J., Langslet, G., Hovingh, G. K., et al. (2015). Anacetrapib as lipid-modifying therapy in patients with heterozygous familial hypercholesterolaemia (REALIZE): a randomized, double-blind, placebo-controlled, phase 3 study. Lancet 385, 2153-2161. doi: 10.1016/S0140-6736(14)62115-2 
Keene, D., Price, C., Shun-Shin, M. J., and Francis, D. P. (2014). Effect on cardiovascular risk of high density lipoprotein targeted drug treatments niacin, fibrates, and CETP inhibitors: meta-analysis of randomised controlled trials including 117411 patients. BMJ 349:g4379. doi: 10.1136/bmj. g4379

Kühnast, S., van der Tuin, S. J., van der Hoorn, J. W., van Klinken, J. B., Simic, B., Pieterman, E., et al. (2015). Anacetrapib reduces progression of atherosclerosis, mainly by reducing non-HDL cholesterol, improves lesion stability and adds to the beneficial effects of atorvastatin. Eur. Heart J. 36, 39-48. doi: 10.1093/eurheartj/ehu319

Liu, M., Chen, Y., Zhang, L., Wang, Q., Ma, X., Li, X., et al. (2015). Regulation of hepatic cholesteryl ester protein expression and reverse cholesterol transport by inhibition of DNA topoisomerase II. J. Biol. Chem. 290, 14418-14429. doi: 10.1074/jbc.m115.643015

Lu, J., Cleary, Y., Maugeais, C., Weber, C. K., and Mazer, N. A. (2015). Analysis of "on/off" kinetics of a CETP inhibitor using a mechanistic model of lipoprotein metabolism and kinetics. CPT Pharmacometrics Syst. Pharmacol. doi: 10.1002/psp4.27. [Epub ahead of print].

Miller, N. E. (2014). CETP inhibitors: time to think again. F1000Res. 3, 124. doi: 10.12688/f1000research.4396.1

Nieland, T. J. F., Xu, S., Penman, M., and Krieger, M. (2011). Negatively cooperative binding of high density lipoprotein to the HDL receptor SR-BI. Biochemistry 50, 1818-1830. doi: 10.1021/bi101657j

Niu, W., and Qi, Y. (2015). Circulating cholesteryl ester transfer protein and coronary heart disease. Mendelian randomization analysis. Circ. Cardiovasc. Genet. 8, 114-121. doi: 10.1161/CIRCGENETICS.114.000748

Rahimi, Z., Nourozi-Rad, R., Rahimi, Z., and Parsian, A. (2012). Strong interaction between $\mathrm{T}$ allele of endothelial nitric oxide synthase with $\mathrm{B} 1$ allele of cholesteryl ester transfer protein TaqIB highly elevates the risk of coronary artery disease and type 2 diabetes mellitus. Hum. Genomics 6:20. doi: 10.1186/1479-7364-6-20

Regieli, J. J., Jukema, J. W., Grobbee, D. E., Kastelein, J. J., Kuivenhoven, J. A., Zwinderman, A. H., et al. (2008). CETP genotype predicts increased mortality in statin treated men with proven cardiovascular disease: an adverse pharmacogenetic interaction. Eur. Heart J. 29, 2792-2799. doi: 10.1093/eurheartj/ehn465
Scharnagl, H., Heuschneider, C., Sailer, S., Kleber, M. F., März, W., and Ritsch, A. (2014). Decreased cholesterol efflux capacity in patients with low cholesteryl ester transfer protein levels. Eur. J. Clin. Invest. 44, 395-401. doi: $10.1111 /$ eci. 12248

Soyal, S. M., Sandhofer, A., Hahne, P., Oberkofler, H., Felder, T., Iglseder, B., et al. (2011). Cholesteryl ester transfer protein and hepatic lipase gene polymorphisms: effects on hepatic mRNA levels, plasma lipids and carotid atherosclerosis. Atherosclerosis 216, 374-380. doi: 10.1016/j.atherosclerosis.2011.01.052

Sun, L., Hu, C., Zheng, C., Huang, Z., Lv, Z., Huang, J., et al. (2014). Gene-gene interaction between CETP and APOE polymorphisms confers higher risk for hypertriglyceridemia in oldest-old Chinese women. Exp. Gerontol. 55, 129-133. doi: 10.1016/j.exger.2014.04.003

Verdoia, M., Schaffer, A., Suryapranata, Y., and De Luca, G. (2015). Effect of HDLmodifiers on cardiovascular outcomes: a meta-analysis of randomized trials. Nutr. Metab. Cardiovasc. Dis. 25, 9-23. doi: 10.1016/j.numecd.2014.09.003

Woollett, L. A., and Spady, D. K. (1997). Kinetic parameters for HDL apoprotein AI and cholesteryl ester transport in the hamster. J. Clin. Invest. 99, 1703-1713. doi: 10.1172/JCI119334

Wu, Z., Lou, Y., Qiu, X., Liu, Y., Lu, L., Chen, Q., et al. (2014). Association of cholesteryl ester transfer protein (CETP) gene polymorphism, high density lipoprotein cholesterol and risk of coronary artery disease; a meta-analysis using a mendelian randomization approach. BMC Med. Genet. 15:118. doi: $10.1186 / \mathrm{s} 12881-014-0118-1$

Conflict of Interest Statement: The author declares that the research was conducted in the absence of any commercial or financial relationships that could be construed as a potential conflict of interest.

Copyright (๑) 2015 Miller. This is an open-access article distributed under the terms of the Creative Commons Attribution License (CC BY). The use, distribution or reproduction in other forums is permitted, provided the original author $(s)$ or licensor are credited and that the original publication in this journal is cited, in accordance with accepted academic practice. No use, distribution or reproduction is permitted which does not comply with these terms. 